\title{
Avaliação dos fatores de risco da entorse em inversão em futebolistas brasileiros e sul-coreanos
}

\author{
Evaluation of risk factors for inversion sprain in Brazilian and South Korean soccer players
}

\author{
Vitória Alves Teixeira, ${ }^{1,}$ \\ Taís Capobianco Genova ${ }^{2}$ \\ Lígia Maria Estigarríbia Tenguan ${ }^{2}$ \\ Daniel Ferreira Moreira Lobato ${ }^{1}$ \\ Fábio Viadanna Serrão
}

\section{Resumo}

Objetivo: Comparar a sensibilidade proprioceptiva do tornozelo e o torque excêntrico eversor e inversor entre jogadores de futebol brasileiros e sul-coreanos, bem como verificar a sua relação com a dominância de membros. Métodos: Participaram deste estudo 14 jogadores de futebol (14-18 anos), divididos em dois grupos: GB- sete indivíduos brasileiros e GSC- sete indivíduos sul-coreanos. A avaliação da sensibilidade proprioceptiva [percepção da posição articular durante os movimentos passivo (PPA-P) e ativo (PPA-A) e limiar de detecção do movimento passivo (LDMP)] foi realizada no dinamômetro isocinético Biodex Multi Joint System 3, a 2\%/s, nos ângulos-alvo de $5^{\circ}$ e $30^{\circ}$ de inversão, e de $5^{\circ}$ e $15^{\circ}$ de eversão. O torque excêntrico foi avaliado no mesmo equipamento, nas velocidades angulares de 30\% e 120\%/s. Resultados: Foi observada diferença na PPA-P (30 de inversão - membro não-dominante $-p=0,001$ ), com o GSC apresentando menores erros, bem como para o pico de torque excêntrico inversor $(30 \% \mathrm{~s}$ - membro dominante - $p=0,02)$, com o GB apresentando maior torque. Demais variáveis apresentaram tendências de menores erros para o GSC e maiores torques para o GB. Conclusões: Apesar das nacionalidades distintas, os dois grupos apresentam características semelhantes quanto à propriocepção do tornozelo e proporção do torque eversor/inversor. Além disso, essas variáveis não foram influenciadas pela dominância de membros.
\end{abstract}

Palavras-Chave: propriocepção, torque, entorses.

\begin{abstract}
Objective To compare the ankle proprioceptive sensitivity and the eccentric eversion and inversion torques between Brazilian and South Korean soccer players, as well as to verify their relation with limb dominance. Methods: Fourteen soccer players (14-18 years old) were divided into two groups: BG seven Brazilian individuals and SKG - seven South Korean individuals. The proprioceptive sensitivity evaluation [joint position sense during passive (JPS-P) and active (JPS-A) movements and threshold to detect passive movement (TTDPM)] was performed on the Biodex MultiJoint System 3 isokinetic dynamometer at $2 \% / \mathrm{s}$, at $5^{\circ}$ and $30^{\circ}$ of ankle inversion, and at 5을 The eccentric torque was evaluated in the same equipment at angular velocities of $30 \% \mathrm{~s}$ and $120 \%$ s. Results: A significant difference was observed for the JPS-P (30 of ankle inversion in non-dominant $\operatorname{limb}-p=0.001$ ), with the SKG presenting smaller erros, as well as for the eccentric inversor peak torque $(30 \% \mathrm{~s}$ - dominant limb - $p=0.02)$, with the BG presenting higher torque. Other variables presented tendencies to lower errors for the SKG and higher torques for GB. Conclusion: Despite the different nationalities, the two groups presented similar characteristics regarding ankle proprioception and eversion/inversion torque ratio. In addition, these variables were not influenced by acute limb dominance.
\end{abstract}

Keywords: proprioception, torque, sprains.
Afiliação dos autores

${ }^{1}$ Universidade Federal do Triângulo Mineiro, Uberaba, Minas Gerais, Brasil.

${ }^{2}$ Universidade Federal de São Carlos, São Carlos, São Paulo, Brasil.

${ }^{*}$ Autor correspondente

Departamento de Fisioterapia Aplicada, Rua Vigário Carlos, 100, Bloco B, 4 andar, Sala 406, Abadia CEP: 38025-350, Uberaba, Minas Gerais, Brasil. e-mail: vitorialvest@gmail.com

Conflito de interesses

Os autores declararam não haver conflito de interesses.

Processo de arbitragem

Recebido: 27/12/2018 Aprovado: 02/03/2019 


\section{Introdução}

As lesões ligamentares do tornozelo apresentam uma ocorrência relativamente alta durante a prática esportiva, especialmente em modalidades que envolvem corridas e saltos, tais como o futebol $^{1,2}$. Estima-se que cerca de $50 \%$ das lesões esportivas estejam relacionadas às entorses laterais do tornozelo ${ }^{3}$, que correspondem a $85 \%$ do total das entorses desta articulação ${ }^{4}$

As lesões ligamentares do tornozelo são, em geral, fenômenos multifatoriais, apresentando fatores de risco extrínsecos e intrínsecos ${ }^{5}$. Em relação aos últimos, algumas possibilidades foram retrospectivamente associadas, tais como a influência da dominância de membros, a diminuição da força muscular, o atraso no tempo de reação muscular e a diminuição do controle postural e da sensibilidade proprioceptiva ${ }^{6,7}$. Ainda existem controvérsias em relação ao teor dessas associações ${ }^{5}$, no entanto, a adequada habilidade proprioceptiva vem sendo considerada como um preditor para o sucesso esportivo e como um fator que diminui o risco de lesão no tornozelo ${ }^{8}$.

Uma das concepções mais defendidas atualmente consiste no fato de que, para minimizar a incidência de um determinado tipo de lesão, deve-se estudar os fatores de risco que podem predispor o atleta ao trauma ${ }^{9}$. Particularmente em relação aos jogadores de futebol, 0 ato de tirar a bola do adversário, por exemplo, envolve forças mediais e laterais que apresentam correspondência com uma maior predisposição à inversão do tornozelo, evidenciando o significativo fator de risco para uma lesão ${ }^{10}$, especialmente no membro dominante, que sofre maior demanda funcional nessas atividades ${ }^{6,7}$.

Além disso, outros movimentos, envolvendo a corrida, a aceleração, a desaceleração, os saltos e suas respectivas aterrissagens; apresentam-se como potenciais desencadeadores de novas lesões ${ }^{1,2}$. Neste sentido, para evitar que uma perturbação externa resulte em uma lesão, os mecanismos neuromusculares devem estar íntegros ${ }^{10}$, assim como a habilidade proprioceptiva, que depende do input neural dos mecanorreceptores localizados nos músculos, ligamentos, cápsulas articulares, tendões e pele, para o Sistema Nervoso Central ${ }^{11}$.

Deste modo, a acuidade da propriocepção fornece informações essenciais para permitir o ajuste das posições e movimentos do tornozelo e parte superior do corpo, a fim de realizar com sucesso o complexo de tarefas motoras necessárias no esporte ${ }^{12}$. Sabe-se que os ligamentos são amplamente solicitados para fornecer informação proprioceptiva e estabilidade para a articulação, e que o recorrente trauma articular por entorses leva à perda da cinemática normal do tornozelo, déficit proprioceptivo e instabilidade funcional da articulação $^{13}$, afetando o desempenho nas atividades esportivas e da vida diária comum.

Da mesma forma, quando se analisa a função muscular no mecanismo de entorse em inversão do tornozelo, constata-se que a contração excêntrica dos músculos eversores e o equilíbrio de força entre esses músculos e os inversores têm uma participação fundamental na sua prevenção, em razão de suas contribuições na desaceleração desse movimento e para impedir a sobrecarga articular ${ }^{14}$. Estudos demonstraram a fraqueza excêntrica dos músculos eversores em pacientes com instabilidade funcional do tornozelo ${ }^{14,15}$, associando esta condição à redução da proteção articular durante o trauma em inversão ${ }^{15}$. Diante disto, a avaliação da proporção do torque excêntrico eversor/inversor tem sido fortemente indicada quando se deseja estudar a importância da força muscular como um fator de risco intrínseco para este tipo de lesão ${ }^{16}$.

No entanto, poucos estudos avaliaram a função muscular excêntrica relacionada ao tornozelo, bem como o seu papel na estabilização dinâmica dessa articulação ${ }^{14,17}$, ou ainda as contribuições proprioceptivas envolvidas nesse mecanismo ${ }^{11,18}$. Desses, há um número ainda menor de estudos que tenham considerado a influência da dominância de membros sobre tais condições $^{19}$. Além disso, nenhum estudo se dedicou à comparação desses fatores em indivíduos de diferentes nacionalidades, que pratiquem o futebol como modalidade de atividade física. Supõe-se que a nacionalidade deva ser considerada quando no estudo dos fatores de risco para as entorses em inversão do tornozelo.

Nesse sentido, este trabalho teve por objetivo comparar a percepção da posição articular (para os movimentos de inversão e eversão do tornozelo) durante o movimento passivo e ativo, bem como o limiar de detecção do movimento passivo e a proporção do torque eversor/inversor excêntrico da articulação do tornozelo entre jogadores de futebol brasileiros e sulcoreanos. Além disso, também foi objetivo do presente estudo investigar a influência da dominância de membros sobre essas variáveis.

\section{Métodos}

\section{Participantes}

Participaram deste estudo transversal 14 homens com idade entre 14 a 18 anos, sadios, jogadores de futebol do Clube Atlético Paulistinha, divididos em dois grupos, de acordo com a sua nacionalidade: GB - composto por sete indivíduos de nacionalidade brasileira (16,4 $\pm 0,5$ anos) e GSC - composto por

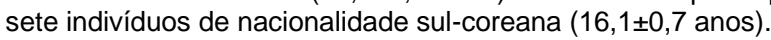

Como critérios de inclusão, os sujeitos deveriam apresentar um tempo mínimo de três anos de prática do futebol, com freqüência mínima de duas horas de treino/dia. Como critérios de não-inclusão, os mesmos não deveriam apresentar episódio prévio de entorse de tornozelo, bem como histórico de disfunção de origem neurológica, cardiovascular, metabólica, reumática e/ou vestibular.

Os responsáveis pelos voluntários assinaram um Termo de Consentimento Livre e Esclarecido, de acordo com a Resolução 196/96 do Conselho Nacional de Saúde. A pesquisa foi aprovada pelo Comitê de Ética em Pesquisa da Universidade Federal de São Carlos (parecer no 211/2005) e conduzida no ano de 2006.

\section{Procedimentos}

Os grupos foram avaliados em apenas um momento distinto, sendo todas as avaliações realizadas bilateralmente. $\mathrm{O}$ membro dominante foi determinado como aquele utilizado preferencialmente pelo voluntário para chutar uma bola durante a sua prática esportiva ${ }^{20}$

\section{Avaliação da sensibilidade proprioceptiva}

A avaliação da percepção da posição articular (PPA) e do limiar de detecção do movimento passivo (LDMP) foi realizada com o voluntário sentado na cadeira do dinamômetro isocinético Biodex Multi-Joint System 3 (Biodex Medical Systems, Inc., Shirley, NY, USA). O encosto da cadeira foi posicionado em uma inclinação de $110^{\circ}$ em relação ao assento e a articulação do joelho foi posicionada em flexão de $45^{\circ}$. Devido à inclinação particular do eixo de inversão-eversão do tornozelo, o eixo mecânico de rotação (eixo mecânico do braço de alavanca) foi alinhado, através de uma aproximação ao eixo de rotação articular, à borda superior do maléolo lateral ${ }^{21}$.

O voluntário permaneceu descalço, com a planta do pé apoiada e o pé estabilizado (por meio de uma faixa de velcro) à plataforma de resistência do dinamômetro, com a articulação do tornozelo em $15^{\circ}$ de flexão plantar $^{22}$ (Figura 1). Para a diminuição das aferências cutâneas neste segmento ${ }^{23}$, foram utilizadas espumas, envolvendo todo o complexo do pé e do tornozelo.

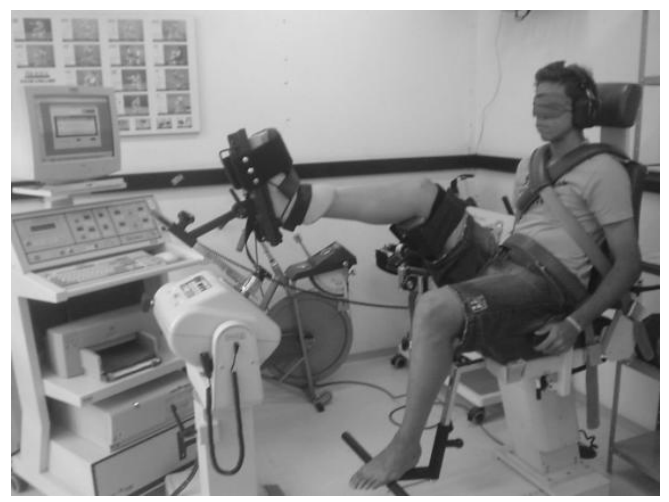

Figura 1. Demonstração do posicionamento utilizado para a avaliação da sensibilidade proprioceptiva. 
O equipamento foi ajustado para mover o pé do voluntário em uma velocidade angular de $2 \%$ s. Os ângulos de inversão e eversão pré-determinados (ângulos-alvo) para a avaliação passiva e ativa da PPA e do LDMP foram: $5^{\circ}$ e $30^{\circ}$ de inversão, e $5^{\circ}$ e $15^{\circ}$ de eversão. Cada ângulo-alvo foi testado três vezes. A ordem dos membros inferiores avaliados, bem como a dos testes proprioceptivos e da direção do movimento (inversão e eversão), foram determinados aleatoriamente por sorteio, para cada voluntário.

Para a avaliação da percepção da posição articular durante o movimento passivo (PPA-P), a articulação do tornozelo foi movida passivamente, a $2 \%$, a partir da posição inicial $\left(0^{\circ}\right.$ posição neutra para a inversão/eversão), em movimentos de inversão e/ou eversão do tornozelo. Ao alcançar o ângulo-alvo de teste, o movimento foi interrompido pelo examinador e a articulação foi mantida nesta posição durante 15 segundos. Após esse período, a articulação retornou à posição inicial, permanecendo nesta posição por mais 15 segundos. Em seguida, o braço de alavanca do dinamômetro novamente movimentou, de forma passiva, a articulação do tornozelo, e o voluntário foi instruído previamente a interromper esse movimento ao identificar o ângulo-alvo, por meio do acionamento de um dispositivo de parada. $\mathrm{O}$ ângulo obtido pelo voluntário durante a realização do teste foi considerado como ângulo de resposta.

A avaliação da percepção da posição da articulação do tornozelo durante o movimento ativo (PPA-A) foi similar à descrita para o movimento passivo, exceto que 0 reposicionamento da articulação do tornozelo foi realizado ativamente pelo voluntário, o qual acionou o dispositivo de parada ao identificar esse ângulo. Para a realização dos testes de PPA, foi utilizada uma máscara para vendar os olhos dos voluntários, cuja finalidade era evitar informação visual a respeito do posicionamento do tornozelo ${ }^{24}$.

Em relação à avaliação do LDMP, a articulação do tornozelo foi movimentada passivamente, na velocidade angular e a partir dos ângulos pré-determinados já descritos, em direção à inversão e/ou eversão, com o voluntário interrompendo o movimento do braço de alavanca do dinamômetro logo que detectasse qualquer alteração na posição articular. Para este teste, além da máscara para vendar os olhos, os voluntários também utilizaram um fone de ouvido para evitar interferências de informações auditivas, durante a execução do mesmo ${ }^{23}$.

Para os testes de PPA, foi utilizada como referência de desempenho dos voluntários a média do erro absoluto (em graus) para cada ângulo-alvo de teste ${ }^{25}$. De forma semelhante, para o LDMP foi utilizada como referência de desempenho a média dos intervalos (em graus) decorridos entre os ângulos de teste e de resposta.

\section{Avaliação do torque excêntrico}

A avaliação do torque excêntrico foi realizada com o voluntário adotando o mesmo posicionamento utilizado para a avaliação proprioceptiva. O voluntário foi inicialmente submetido a um procedimento de familiarização com o equipamento e com a velocidade a ser utilizada, por meio de cinco $(30 \% / \mathrm{s})$ ou 10 $(120 \%$ s) contrações sub-máximas de inversão e eversão excêntricas do tornozelo. Após esse procedimento, houve um descanso de 60 segundos antes de se iniciar a avaliação propriamente dita.
A amplitude de movimento para o teste foi controlada em $45^{\circ}$ ( $30^{\circ}$ para a inversão e $15^{\circ}$ para a eversão). O primeiro teste consistiu de cinco contrações excêntricas máximas dos músculos eversores e inversores do tornozelo a uma velocidade de $30 \% \mathrm{~s}$, e o segundo, de 10 contrações de mesma característica a uma velocidade de $120 \%$ s, sendo fornecido um intervalo de 120 segundos entre os dois testes (Figura 2).

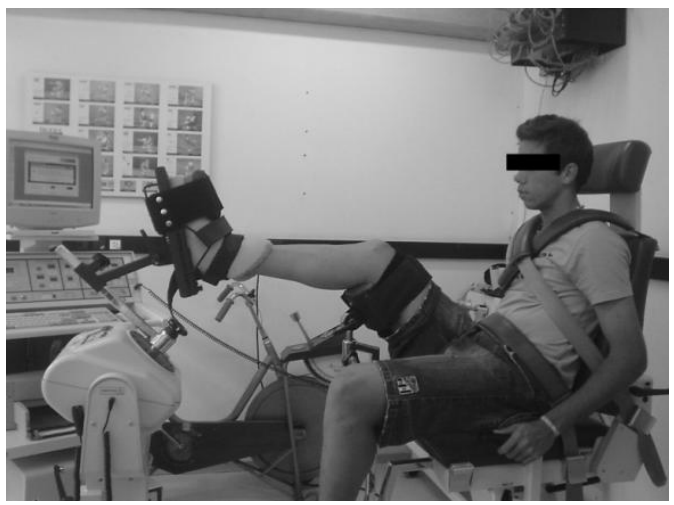

Figura 2. Demonstração do posicionamento utilizado para a avaliação do torque excêntrico.

As variáveis isocinéticas utilizadas no presente estudo foram a média da razão pico de torque excêntrico por peso corporal, tanto para os movimentos de inversão quanto de eversão do tornozelo, bem como a média entre a proporção do torque eversor/inversor excêntrico (relação agonista/antagonista)

\section{Análise dos dados}

Para a análise dos dados foi utilizado o programa STATISTICA 5.0 for Windows (StatSoft). As medidas referentes à sensibilidade proprioceptiva e ao torque foram analisadas através da estatística descritiva (média e desvio-padrão) e, de acordo com as características das distribuições de seus dados (verificada pelo teste de Shapiro Wilks), comparadas pelos testes t-Student para amostras dependentes e de Wilcoxon (análise intragrupos), bem como pelos testes t-Student para amostras independentes e $U$ de Mann-Whitney (análise intergrupos), para cada ângulo-alvo de inversão (5 e 30 graus) e eversão (5 e 15 graus) do tornozelo, considerando um nível de significância de $5 \%$.

\section{Resultados}

Não foram encontradas diferenças entre os grupos quanto à idade $(p=0,40)$, estatura $(p=0,17)$, massa corporal $(p=0,18)$ e índice de massa corporal $(p=0,51)$. Em relação às avaliações proprioceptivas, foi observada diferença intergrupos significativa apenas para $o$ teste de PPA-P $(p=0,001)$, quando comparado o membro não-dominante no ângulo de $30^{\circ}$ de inversão, com o GSC apresentando os menores erros (Tabela 1). As comparações intragrupos realizadas não evidenciaram diferenças significativas na acuidade proprioceptiva entre os membros dominante (MDOM) e não-dominante (MNDOM).

\section{Tabela 1}

Média e desvio-padrão do erro absoluto nos testes de PPA-P e PPA-A e do intervalo de erro no teste de LDMP dos MDOM e MNDOM do GB e GSC $\mathrm{n}=07$.

\begin{tabular}{|c|c|c|c|c|c|c|c|c|}
\hline & \multicolumn{4}{|c|}{ GB } & \multicolumn{4}{|c|}{ GSC } \\
\hline & \multicolumn{2}{|c|}{ MDOM } & \multicolumn{2}{|c|}{ MNDOM } & \multicolumn{2}{|c|}{ MDOM } & \multicolumn{2}{|c|}{ MNDOM } \\
\hline EV & $5^{\circ}$ & $15^{0}$ & $5^{0}$ & $15^{\circ}$ & $5^{0}$ & $15^{0}$ & $5^{0}$ & $15^{\circ}$ \\
\hline PPA-P & $1,6^{\circ} \pm 0,8^{\circ}$ & $2,7^{\circ} \pm 2,1^{\circ}$ & $0,8^{\circ} \pm 1,1^{\circ}$ & $1,6^{\circ} \pm 1,4^{\circ}$ & $1,1^{\circ} \pm 1,0^{\circ}$ & $3,1^{\circ} \pm 2,3^{\circ}$ & $1,1^{\circ} \pm 1,2^{\circ}$ & $2,3^{\circ} \pm 1,3^{\circ}$ \\
\hline PPA-A & $3,8^{\circ} \pm 2,5^{\circ}$ & $3,9^{\circ} \pm 2,4^{\circ}$ & $2,7^{\circ} \pm 1,9^{\circ}$ & $2,9^{\circ} \pm 2,1^{\circ}$ & $2,8^{\circ} \pm 2,9^{\circ}$ & $2,9^{\circ} \pm 1,9^{\circ}$ & $4,3^{\circ} \pm 4,3^{\circ}$ & $4,3^{\circ} \pm 3,2^{\circ}$ \\
\hline LDMP & $1,5^{\circ} \pm 1,2^{\circ}$ & $1,9^{\circ} \pm 2,1^{\circ}$ & $1,0^{\circ} \pm 0,7^{\circ}$ & $1,1^{\circ} \pm 1,2^{\circ}$ & $0,9^{\circ} \pm 0,9^{\circ}$ & $1,2^{\circ} \pm 0,9^{\circ}$ & $1,3^{\circ} \pm 1,0^{\circ}$ & $1,2^{\circ} \pm 1,4^{\circ}$ \\
\hline INV & $5^{0}$ & $30^{\circ}$ & $5^{\circ}$ & $30^{\circ}$ & $5^{\circ}$ & $30^{\circ}$ & $5^{\circ}$ & $30^{\circ}$ \\
\hline PPA-P & $1,6^{\circ} \pm 1,8^{\circ}$ & $5,7^{\circ} \pm 2,2^{\circ}$ & $0,7^{\circ} \pm 0,3^{\circ}$ & $6,5^{\circ} \pm 1,1^{\circ}$ & $1,3^{\circ} \pm 1,3^{\circ}$ & $5,7^{\circ} \pm 2,9^{\circ}$ & $1,4^{\circ} \pm 0,8^{\circ}$ & $2,9^{\circ} \pm 1,8^{\circ}$ \\
\hline PPA-A & $4,1^{\circ} \pm 2,6^{\circ}$ & $3,8^{\circ} \pm 2,6^{\circ}$ & $2,8^{\circ} \pm 2,4^{\circ}$ & $4,9^{\circ} \pm 4,6^{\circ}$ & $2,2^{\circ} \pm 1,7^{\circ}$ & $4,1^{\circ} \pm 2,5^{\circ}$ & $3,2^{\circ} \pm 2,1^{\circ}$ & $5,3^{\circ} \pm 2,1^{\circ}$ \\
\hline LDMP & $1,5^{\circ} \pm 1,3^{\circ}$ & $1,5^{\circ} \pm 1,4^{\circ}$ & $1,6^{\circ} \pm 1,5^{\circ}$ & $1,1^{\circ} \pm 0,8^{\circ}$ & $1,0^{\circ} \pm 0,7^{\circ}$ & $0,9^{\circ} \pm 0,8^{\circ}$ & $1,3^{\circ} \pm 0,7^{\circ}$ & $1,3^{\circ} \pm 0,9^{\circ}$ \\
\hline
\end{tabular}

MNDOM: Membro não-dominante; EV: Eversão do tornozelo; INV: inversão do tornozelo; PPA-P: Percepção da posição articular durante o movimento passivo; PPA A: Percepção da posição articular durante o movimento ativo; LDMP - Limiar de detecção do movimento passivo. 
Em relação aos valores de torque, foi observada diferença intergrupos significativa apenas para o pico de torque excêntrico inversor - membro dominante $(p=0,02)$ na velocidade de $30 \%$, com o GB apresentando maior torque (Tabela 2). Na Tabela 2

Média e desvio-padrão do pico de torque excêntrico dos músculos eversores e inversores do tornozelo e da relação entre o pico de torque excêntrico eversor/inversor em cada velocidade, para os MDOM e MNDOM do GB e GSC. n=07.

\begin{tabular}{|c|c|c|c|c|c|c|c|c|}
\hline & \multicolumn{4}{|c|}{ GB } & \multicolumn{4}{|c|}{ GSC } \\
\hline & \multicolumn{2}{|c|}{ MDOM } & \multicolumn{2}{|c|}{ MNDOM } & \multicolumn{2}{|c|}{ MDOM } & \multicolumn{2}{|c|}{ MNDOM } \\
\hline & $30 \% / \mathrm{s}$ & $120 \% / \mathrm{s}$ & $30 \% / \mathrm{s}$ & $120 \% / \mathrm{s}$ & $30 \% / \mathrm{s}$ & $120 \% / \mathrm{s}$ & $30 \% / \mathrm{s}$ & $120 \% \mathrm{~s}$ \\
\hline $\mathrm{EV}(\mathrm{Nm})$ & $59,1 \pm 11,7$ & $56,1 \pm 14,6$ & $60,8 \pm 9,9$ & $59,1 \pm 18,1$ & $48,7 \pm 7,6$ & $48,1 \pm 14,4$ & $49,4 \pm 13,6$ & $47,7 \pm 15,7$ \\
\hline INV (Nm) & $64,1 \pm 10,8$ & $60,0 \pm 12,7$ & $61,9 \pm 12,3$ & $61,9 \pm 18,2$ & $48,4 \pm 11,3^{*}$ & $48,9 \pm 11,4$ & $48,2 \pm 14,4$ & $47,3 \pm 13,9$ \\
\hline EV/INV & $91,9 \pm 3,2$ & $93,0 \pm 11,2$ & $99,2 \pm 8,3$ & $94,9 \pm 3,2$ & $102,8 \pm 18,4$ & $98,8 \pm 14,5$ & $104,7 \pm 21,8$ & $100,3 \pm 14,5$ \\
\hline
\end{tabular}

MNDOM: Membro não-dominante; EV: Torque eversor do tornozelo; INV: torque inversor do tornozelo; EV/INV: Relação eversor/inversor do tornozelo.

Não houve diferença entre os grupos para a relação pico de torque excêntrico eversor/inversor, apenas tendências de menores relações para o $G B(p=0,15-0,85)$. Na comparação intragrupos (MDOM X MNDOM), não foi observada diferença significativa, apenas tendências de menor relação para o MDOM $(\mathrm{p}=0,08-0,81)$, Tabela 2 .

\section{Discussão}

A acuidade proprioceptiva do tornozelo não foi estudada tão extensivamente em situações de instabilidade articular quanto nos casos de instabilidade do joelho ${ }^{11} \mathrm{e}$, considerando a situação de integridade articular desta articulação, o número de trabalhos que utilizou esse tipo de avaliação é ainda mais escasso ${ }^{18,25}$. Além disso, os resultados desses estudos ainda não esclareceram o tema, devido à ausência de evidências que suportem os achados observados.

Com relação aos resultados do teste de PPA-P, foi encontrada diferença significativa entre os grupos em relação ao ângulo-alvo de $30^{\circ}$ de inversão no MNDOM, com o GSC apresentando os menores erros. Houve tendência de melhor desempenho dos jogadores sul-coreanos para os ângulos relacionados à inversão do tornozelo, enquanto os jogadores brasileiros apresentaram tendência de melhor desempenho para os ângulos relacionados à eversão.

Em conjunto, tais achados poderiam revelar, em um primeiro momento, melhor desempenho proprioceptivo em inversão para os jogadores sul-coreanos, permitindo estratégias mais eficientes de controle desse movimento e minimizando as chances de ocorrência de entorses laterais nesse grupo, que caracteristicamente ocorrem no arco compreendido entre 0-30 de inversão ${ }^{14}$. Tais resultados recebem suporte de De Noronha et al. $(2006)^{25}$, que apontam o desempenho no teste de PPA-P como um importante fator preditivo de entorses laterais do tornozelo. Entretanto, como a maioria das diferenças intergrupos não atingiu a significância estatística mínima, deve-se ter cautela na interpretação desses resultados, assim como para aqueles relacionados ao MDOM, onde o GSC apresentou tendência de menores erros absolutos (PPA-A e PPA-P), na maioria das situações avaliadas, também sem significância estatística.

$\mathrm{Na}$ comparação entre membros, os jogadores sul-coreanos apresentaram tendência de melhor desempenho (LDMP e PPAA) para o MDOM, quando comparado ao contralateral, enquanto os jogadores brasileiros apresentaram tendência contrária. Considerando a maior demanda funcional que o MDOM sofre durante as atividades esportivas ${ }^{9}$, e que a integridade da propriocepção é um dos componentes necessários para a estabilização articular durante a prática dessas atividades, infere-se que os jogadores de futebol sul-coreanos, avaliados por este estudo podem apresentar menor risco de entorse lateral do tornozelo no MDOM do que no membro contralateral e que o inverso possa ocorrer para os jogadores brasileiros, segundo (apenas) o critério de contribuição proprioceptiva.

$\mathrm{Em}$ relação às variáveis isocinéticas, foram observadas diferenças significativas no torque inversor (MDOM) apenas na velocidade de 30\%, com o GB apresentando maior torque. Embora não significativo, também foi verificado melhor desempenho para os jogadores brasileiros, em todas as demais situações analisadas. Tal superioridade não significa, porém, benefícios para o grupo de jogadores brasileiros, ou prejuízos para o grupo de jogadores sul-coreanos. Embora alguns estudos apontem associações entre a fraqueza excêntrica dos músculos eversores do tornozelo e os episódios de instabilidade e de lesões desta articulação $0^{14,15,19}$, o desequilíbrio de forças comparação intragrupos (MDOM X MNDOM), não foi observada diferença significativa ou tendência de melhor desempenho para um determinado membro. musculares (agonista/antagonista), representado por alterações na relação eversor/inversor, parece apresentar maior importância como um dos fatores de risco intrínsecos para a ocorrência das entorses laterais ${ }^{7,26,27}$.

Neste sentido, a comparação entre os grupos para essa relação evidenciou que, para ambas as velocidades angulares, os jogadores brasileiros apresentaram predominância de torque do grupo inversor sobre o eversor, caracterizado por proporções eversores/inversores com magnitudes inferiores a $100(1,00)$, enquanto os jogadores sul-coreanos apresentaram predominância do grupo eversor sobre o inversor (exceto para o MDOM, a $120 \%$ s), caracterizado por proporções eversores/inversores com magnitudes superiores a $100(1,00)$. Nickson $^{28}$ indica valores normativos de $0,79(30 \%$ s) e 0,74 $(120 \% / \mathrm{s})$ para a relação eversores/inversores do tornozelo, enquanto Leslie et al. ${ }^{16}$ apontam relações ótimas de 0,80 (30\%/s) e $0,63(120 \%)$, portanto muito inferiores às observadas no presente estudo. Neste caso, os jogadores sul-coreanos, especialmente, estariam mais expostos às entorses articulares, analisando unicamente por este critério

É possível que diferenças biológicas ou relacionadas a treinamentos prévios tenham alguma influência sobre tais resultados. No entanto, ambos os grupos apresentaram relações eversores/inversores elevadas (especialmente o GSC), sendo esta alteração indesejável para uma estabilidade dinâmica adequada da articulação do tornozelo, bem como caracterizando um possível fator de risco para a entorse lateral desta articulação ${ }^{26,27}$. Entretanto, cabe ressaltar que os valores normativos citados se referem ao teste isocinético concêntrico, e que diferenças importantes podem existir em relação à modalidade excêntrica, a qual ainda não apresenta valores de referência.

Em relação à dominância de membros, Ekstrand \& Gillquist ${ }^{6}$ notaram que o MDOM sustentou mais lesões no tornozelo (comparativamente ao membro contralateral) em jogadores de futebol, apresentando cerca de $92 \%$ do total de lesões desta articulação. No entanto, ainda não está completamente estabelecida a forma como a mesma se relaciona com uma maior predisposição a lesões, exceto pela maior demanda funcional ao qual o MDOM está exposto ${ }^{9}$. Neste sentido, a associação entre o risco de lesões e a dominância de membros pode ser analisada pelo fato deste membro ser o mais usado para as atividades esportivas, especialmente para aquelas que envolvem o chute, o salto e a aterrissagem ${ }^{22}$

No presente estudo, não foi verificada influência da dominância de membros sobre as variáveis analisadas. Tal fato aponta para a ausência de assimetrias proprioceptivas importantes entre os MDOM e MNDOM, o que já foi sugerido previamente para outros complexos articulares ${ }^{29}$ ou mesmo para o tornozelo ${ }^{30}$. Quanto à avaliação do torque, não está descartada a possibilidade de que as características da relação eversor/inversor possam ser influenciadas pela dominância de membros, uma vez que o GB apresentou maior relação para o MNDOM em relação ao MDOM, na velocidade de 30\%/s.

No entanto, tais hipóteses são preliminares e, considerando o reduzido tamanho da amostra utilizada, torna-se difícil realizar inferências para a população de jogadores brasileiros e sulcoreanos, em geral. Ainda que esse número fosse superior, diferenças quanto a fatores relacionados à idade, estatura, massa corporal, tipo de treinamento, tempo de atuação, dentre outros, poderiam repercutir de forma variável nas características proprioceptivas $e$ isocinéticas, sendo difícil realizar tais generalizações. Contudo, para o presente estudo, cuidados foram tomados no sentido garantir a homogeneidade das 
amostras quanto às características acima descritas, a fim de garantir maior suporte aos nossos resultados.

O desenvolvimento de programas de tratamento e prevenção das entroses em inversão do tornozelo está intimamente relacionado à identificação desses elementos, sendo a propriocepção e a força muscular apontadas como dois de seus possíveis fatores intrínsecos ${ }^{7,25,27}$. Embora o presente estudo não tenha encontrado evidências sólidas a respeito da identificação desses fatores, o mesmo indica a necessidade da realização de novos trabalhos, incluindo os de natureza prospectiva, que possam associar-se ao conhecimento já existente sobre $\mathrm{o}$ assunto, e melhor direcionar a conduta terapêutica desses programas.

\section{Conclusão}

Apesar das nacionalidades distintas, os dois grupos apresentam características semelhantes quanto à propriocepção do tornozelo e proporção do torque eversor/inversor. Os dois grupos apresentaram relações eversores/inversores elevadas, o que os posiciona de forma similar quanto aos fatores de risco analisados por este estudo. Além disso, essas variáveis não foram influenciadas pela dominância de membros.

\section{Agradecimentos}

Agradecemos ao Clube Atlético Paulistinha pela parceria e por viabilizar o contato com os atletas participantes do estudo.

\section{Referências}

1. Garrick JG, Requa RK. The epidemiology of foot and ankle injuries in sports. Clin Podiatr Med Surg 1989; 6:629-637.

2. Hickey GJ, Ffricker PA, McDonald WA. Injuries of young elite female basketball players over a six-year period. Clin J Sport Med 1997; 7(4):252256.

3. Szczerba JE, Bernier JN, Perrin DH, Gansneder BM. Intertester reliability of active and passive ankle joint position sense testing. J Sport Rehabil $1995 ; 4: 282-291$

4. van den Bekerom MP, Kerkhoffs GM, McCollum GA, Calder JD, van Dijk $\mathrm{CN}$. Management of acute lateral ankle ligament injury in the athlete. Knee Surg Sports Traumatol Arthrosc 2013; 21(6):1390-1395.

5. Murphy DF, Connolly DAJ, Beynnon BD. Risk factors for lower extremity injuries: a review of the literature. Br J Sports Med 2003; 37(1):13-29.

6. Ekstrand J, Gillquist J. Soccer injuries and their mechanisms: a prospective study. Med Sci Sports Exerc 1983; 15(3):267-270.

7. Willems TM, Witvrouw E, Delbaere K, Mahieu N, DE Bourdeaudhuig I, DE Clercq D. Intrinsic risk factors for inversion ankle sprains in male subjects. Am J Sports Med 2005; 33(3):415-423.

8. Witchalls J, Blanch P, Waddington G, Adams R. Intrinsic functional deficits associated with increased risk of ankle injuries: a systematic review with meta-analysis. Br J Sports Med. 2012; 46(7):515-523.

9. Beynnon DB, Murphy DF, Alosa DM. Predictive factors for lateral ankle sprain: A literature review. J Athl Train 2002; 37(4):376-380.
10. Giza E, Fuller C, Junge A, Dvorak J. Mechanisms of foot and ankle injuries in soccer. Am J Sports Med 2003; 31(4):550-554.

11. Steinberg N, Adams R, Ayalon M, Dotan N, Bretter S, Waddington G. Recent ankle injury, sport participation level and tests of proprioception. J Sport Rehabil 2018; 9:1-25

12. Di Giulio I, Maganaris CN, Baltzopoulos V, Loram ID. The proprioceptive and agonist roles of gastrocnemius, soleus and tibialis anterior muscles in maintaining human upright posture. J Physiol, 2009; 587(10):2399-2416.

13. Anandacoomarasamy A, Barnsley L. Long-term outcomes of inversion ankle injuries. Br J Sports Med 2005; 39:e14 [discussion: e14].

14. Yildiz Y, Aydin T, Sekir U, Hazneci B, Komurcu M, Kaylon TA. Peak end range eccentric evertor/concentric invertor muscle strength ratios in chronically instable ankles: comparison with healthy individuals. J Sports Sci Med 2003; 2(3):70-76.

15. Willems T, Witvrouw E, Verstuyft J, Vaes P, De Clercq D. Proprioception and muscle strength in subjects with a history of ankle sprains and chronic instability. J Athl Train 2002; 37(4):487-493.

16. Leslie M, Zachazewski J, Browne B. Reability of isokinetic torque values for ankle invertors and evertors. J Orthop Sports Phys Ther 1990; 11(12):612 616.

17. Wisthoff B, Matheny S, Struminger A, Gustavsen G, Glutting J, Swanik C, Kaminski TW. Ankle Strength Deficits in a Cohort of Collegiate Athletes with Chronic Ankle Instability. J Sport Rehabil 2018; 17:1-22.

18. Han J, Anson J, Waddington G, Adams R, Liu Y. The Role of Ankle Proprioception for Balance Control in relation to Sports Performance and Injury. Biomed Res Int 2015; 2015:842804.

19. Lin WH, Liu YF, Hsieh CC, Lee AJ. Ankle eversion to inversion strength ratio and static balance control in the dominant and non-dominant limbs of young adults. J Sci Med Sport 2009; 12(1):42-49.

20. Bolgla LA, Keskula DR. Reliability of lower extremity functional performance tests. J Orthop Sports Phys Ther 1997; 26(3):138-142.

21. Dvir Z. Isokinetics - Muscle Testing, Interpretation and Clinical Applications. Churchil Livingstone: Edinburgh, 1995.

22. Willems TM, Witvrouw E, Delbaere K, Philippaerts R, DE Bourdeaudhuig I, $\mathrm{DE}$ Clercq D. Intrinsic risk factors for inversion ankle sprains in females - A prospective study. Scand J Med Sci Sports 2005; 15(5):336-345.

23. Reider B, Arcand MA, Diehl LH, Mroczek K, Abulencia A, Stroud CC, Palm $M$, Gilbertson J, Staszak P. Proprioception of the knee before and after anterior cruciate ligament reconstruction. Arthroscopy 2003; 19(1):2-12.

24. Barrack RL, Lund PJ, Munn BG, Wink C, Happel L. Evidence of reinnervation of free patellar tendon autograft used for anterior cruciate reinnervation of free patellar tendon autograft used for anterior

25. De Noronha M, Refshauge KM, Herbert RD, Kilbreath SL. Do voluntary strength, proprioception, range of motion, or postural sway predic ocurrence of lateral ankle sprain? Br J Sports Med 2006; 40:824-828.

26. Baumhauer JF, Alosa DM, Renstrom P, Trevino S, Beynnon B. A prospective study of ankle injury risk factors. Am J Sports Med 1995; 23(5):564-570

27. Wang HK, Chen $\mathrm{CH}$, Shiang TY, Jan MH, Lin KH. Risk-Factor Analysis of High School Basketball-Player Ankle Injuries: A Prospective Controlled Cohort Study Evaluating Postural Sway, Ankle Strength, and Flexibility Arch Phys Med Rehabil 2006; 87(6):821-825.

28. Nickson W. Normative isokinetic data on the ankle invertors and evertors. Aust J Physiother 1987; 33:85-90.

29. Aydin T, Yildiz Y, Yanmis I, Yildiz C, Kalyon TA Shoulder proprioception: comparison between the shoulder joint in healthy and surgically repaired shoulders. Arch Orthop Trauma Surg 2001; 121(7):422-425.

30. Fu ASN, Hui-Chan CWY. Ankle joint proprioception and postural control in basketball players with bilateral ankle sprains. Am J Sports Med 2005 33(8):1174-1182. 